\title{
General Guidelines for the Heteroepitaxial Thin Film Growth Established by Combinatorial X-ray Diffraction
}

\author{
Dmitry A. Kunuruznyak and Toyohiro ChiKYow \\ National Institute for Materials Science, Advanced Electronic Materials Center, Advanced Device Materials Group \\ 1-1 Namiki, Tsukuba, Ibaraki 305-0044
}

(Received October 10, 2006)

\begin{abstract}
Heteroepitaxial thin films are widely used in a broad range of modern electronic devices, for example as the highdielectric-constant (high- $k$ ) gates in field effect transistors. In order to improve the crystal quality of epitaxial layers, researchers develop intricate and costly deposition processes. An alternative paradigm should involve deposition of the complex compounds that could exhibit unusual behavior and yield the high quality films by simple and industry-friendly processes. Successful search for the new complex compounds must employ combinatorial methods. In this publication, we demonstrate fabrication of composition spread materials libraries by Combi-PLD and their high-throughput characterization by two different XRD techniques. Generally, the composition spreads have polycrystalline, textured, and epitaxial areas of varied quality. We use statistical analysis to establish general guidelines for the epitaxial growth. Using the NiO$\mathrm{Co}_{3} \mathrm{O}_{4}-\mathrm{Mn}_{2} \mathrm{O}_{3}$ system on $\mathrm{SrTiO}_{3}$ as an example, we demonstrate that for the chemically inert interfaces lattice mismatch is the most important factor of the commensurate growth. For example, the rocksalt-perovskite interface requires the optimal lattice mismatch of $\sim 6.5 \%$. Examination of the $\mathrm{Y}_{2} \mathrm{O}_{3}-\mathrm{HfO}_{2}-\mathrm{Al}_{2} \mathrm{O}_{3}$ system on $\mathrm{Si}(100)$ revealed a narrow epitaxial area comprised of a new metastable crystal phase. Thus, stable epitaxial interface is attainable even for the chemically reactive boundaries. In addition to lattice mismatch, the high-quality heteroepitaxial film requires balancing the reaction with the outdiffusion of the reaction products away from the interface.
\end{abstract}

\section{Introduction}

Heteroepitaxial thin film layers are used in a broad variety of modern electronic applications. For example, such substances as $\mathrm{SiGe}$, gallium nitride, gallium arsenide and indium phosphide are used in semiconductor devices, telecommunications and LEDs..$^{1,2)}$ Multiferroic materials ${ }^{3)}$ combine electronic and magnetic properties into multifunctional materials, so that a single device component can perform more than one task. The transverse epitaxial multiferroic nanostructures with controlled morphologies such as $\mathrm{PbTiO}_{3}-\mathrm{CoMn}_{2} \mathrm{O}_{4}$ on $\mathrm{SrTiO}_{3}$ should create strong elastic interactions between the two phases combining the $\mathrm{PbTiO}_{3}$ piezoelectric effect, and large magnetostriction of ferrimagnetic $\mathrm{CoMn}_{2} \mathrm{O}_{4}{ }^{4)}$ In the above examples thin films are chemically inert in contact with the substrate.

Recently, heteroepitaxial oxide thin films on silicon attract much attention because of their importance as gate insulator materials in field effect transis-

E-mail: Dmitry.kukuruznyak@nims.go.jp tors (FETs). ${ }^{5)}$ The ability to continue scaling microelectronic devices to ever smaller dimensions quantified by Moor's law is limited by quantum mechanical effects. $\left.{ }^{6}\right)$ As the thickness of conventional silicon dioxide gates is reduced to just a few atomic layers, electrons can tunnel directly through the films. Continued device scaling requires the replacement of the insulator with high-dielectric-constant (high- $k$ ) epitaxial oxides to increase its thickness. ${ }^{7)}$ However, interfacing an ionic oxide with silicon is a major challenge because these two materials chemically react either during deposition or post-deposition treatment. ${ }^{8)}$

Generally, development of the new types of heteroepitaxial thin films for novel applications is a very complicated and laborious process. In a usual approach, researchers first choose bulk materials with known physical properties and then develop an appropriate technique for its fabrication in thin-film form. The combination of the simple materials and complex deposition techniques is very costly and inefficient for commercial mass production. We advocate an alternative and 'industry-friendly' approach. 
We stand by a limited number of the least expensive thin film deposition methods, and seek for new complex multiple-component materials which would yield satisfactory results even for the simplest manufacturing processes. This fabrication-oriented approach requires significant broadening of the standard materials arsenals.

In this new paradigm combinatorial and highthroughput experimentation methods acquire great importance. Fabrication of new material libraries opens up vast uncharted territories of complex systems, and high-throughput tests quickly survey these territories, isolate and characterize substances with desired properties. Fabrication of large materials libraries using thin film techniques has been recently demonstrated. ${ }^{9)}$ These libraries are usually formed as composition spreads, where chemical composition of the films gradually changes in one or two directions. Unfortunately, there is a significant lag in development of suitable characterization and analysis methods for such libraries.

In this publication we describe adaptation of the $\mathrm{X}$-ray diffraction (XRD) methods to combinatorial exploration of composition spread thin films. We employ these methods for systematic studies of the crystal quality of epitaxial films for different materials systems. Heteroepitaxial films can be divided into two general categories: the films which are chemically inert in contact with the substrate (like $\mathrm{GeSi}$ on $\mathrm{Si}$ or $\mathrm{PbTiO}_{3}$ on $\mathrm{SrTiO}_{3}$ ), and the films which react with the substrate either during deposition or during post-deposition processing (like high- $k$ oxides on silicon). We have established that regularities of the epitaxial growth are strongly dependent on the chemical stability of the film-substrate interface. For materials from the first category, (inert interfaces) the most important parameter is the lattice mismatch. For materials from the second category, (reactive interfaces ) chemical reactivity and diffusion of the byproducts are dominating factors.

\section{Experimental}

\section{1 Composition spread thin films}

Combinatorial materials libraries are frequently prepared as composition spread thin films. ${ }^{10)}$ Fabrication of the composition spreads involves spatially varying deposition of materials on a single substrate. Any solid material can be viewed as being composed of atomic layers that are stacked periodically. Some thin film deposition techniques such as molecular beam epitaxy (MBE) or pulsed laser deposition
(PLD) can used to control atomic layering sequencing in the film. ${ }^{11)}$ The composition and sequence of each layer are manipulated by motion of the shadow masks during deposition. Below we describe fabrication of the continuous ternary composition spread samples by combinatorial PLD (Combi-PLD) using an automated shutter and a rotating substrate holder.

The sample consists of three interpenetrating and intermixed stripes of elemental oxides rotated with respect to each other by $120^{\circ}$ as shown in Fig. 1 . Thus, the obtained sample contains a full range of ternary oxide compositions (regular triangular area in the center) as well as three separate full binary oxides spreads. Each stripe is deposited in multiple steps to insure atomic mixing of the cations. First a single wedged stripe of material $\mathrm{A}$ is deposited with the help of a moving shadow mask and pulsed ablation with deposition rate of approximately $\sim 0.05 \AA$ per pulse. The tapered stripe had a thickness ranging from 0 to approximately $5 \AA$. Then the substrate is rotated and the stripe of material $\mathrm{B}$ is deposited in the same way. Finally, the graded-thickness stripe of material $\mathrm{C}$ is deposited. After all three individual wedged stripes are formed the process is repeated to eventually obtain $\sim 100 \mathrm{~nm}$-thick composition spreads. The deposition rate for each material is determined prior to fabrication of the ternary maps, and the number of pulses for forming each stripe is adjusted to obtain the ternary film of a uniform thickness. Details of this deposition technique, along with the description of the Combinatorial PLD equipment, its moving shutters and rotating heated substrate holder are described elsewhere. ${ }^{12)}$

\section{2 Combinatorial-XRD}

The ideal method to determine the crystal structure, texture and orientation of the crystal growth of the different points in the composition spread thin films is combinatorial X-ray diffraction (CombiXRD). We demonstrate these combinatorial XRD measurements using the Bruker AXS D8 Discover with GADDS Super Speed instrument equipped with a Hi-Star two-dimensional detector and a scanning $\mathrm{XY}$ stage.

The diameter of the collimated X-ray beam, as well as the sample scanning step in both $\mathrm{X}$ and $\mathrm{Y}$ directions was $0.3 \mathrm{~mm}$. A rectangle containing ternary diagram is broken up into $27 \times 34=918$ pixels. With such resolution the relative molar fractions of the cations within a single pixel varied by less than $5 \%$. Higher resolution (smaller beam size and scanning step) was possible but yielded unreasonably large 


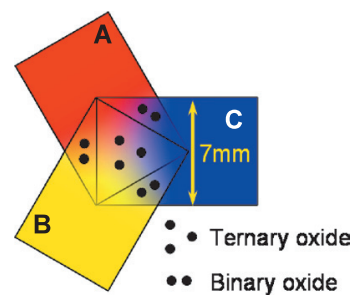

Fig. 1. Schematic representation of the ternary continuous composition spread sample.

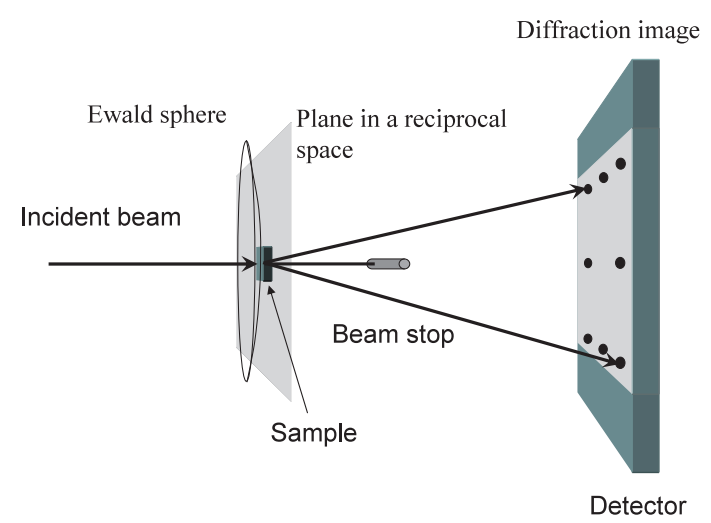

Fig. 2. Sketch of the experimental setup for high energy Xray diffraction in transmission geometry.

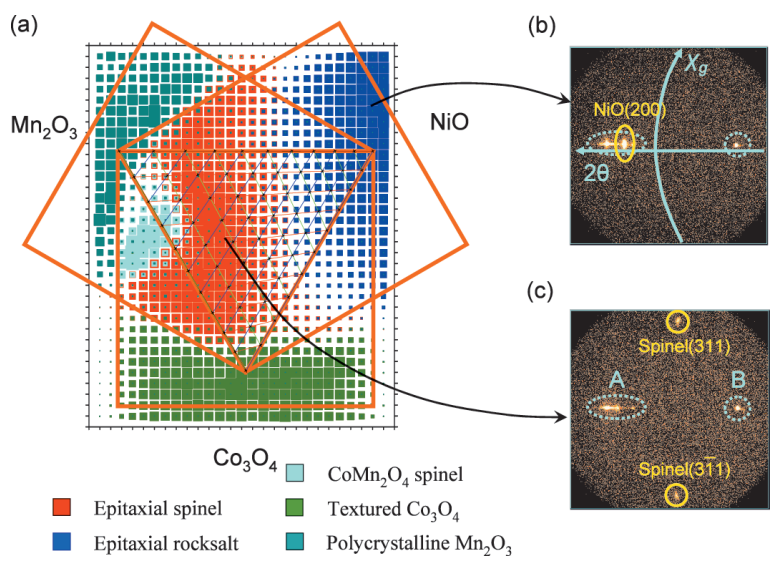

Fig. 3. (a) Reconstruction of the ternary oxide crystal phase diagram for the $\mathrm{NiO}-\mathrm{Co}_{3} \mathrm{O}_{4}-\mathrm{Mn}_{2} \mathrm{O}_{3}$ system deposited onto $\mathrm{SrTiO}_{3}$. (b) The two-dimensional XRD spectrum (frame) at the area of the epitaxial $\mathrm{NiO}$ rocksalt phase. The frame contains diffraction signal for $2 \theta$ in the range between $20^{\circ}$ and $55^{\circ}$, and for the angle $-126^{\circ}<\chi_{\mathrm{g}}<-54^{\circ}$. The observed $\mathrm{NiO}(200)$ diffraction spot corresponds to $2 \theta=43.3^{\circ}$ and $<\chi_{\mathrm{g}}=$ $-90^{\circ}$. (c) The frame at the area of epitaxial spinel. The recorded spinel(311) diffraction spots correspond to $2 \theta=36.3^{\circ}$. Peaks A $\left(2 \theta=46.47^{\circ}\right)$ and B $\left(2 \theta=22.75^{\circ}\right)$ are the $(100)$ and $(200)$ reflections from the $\mathrm{SrTiO}_{3}$ substrate respectively.

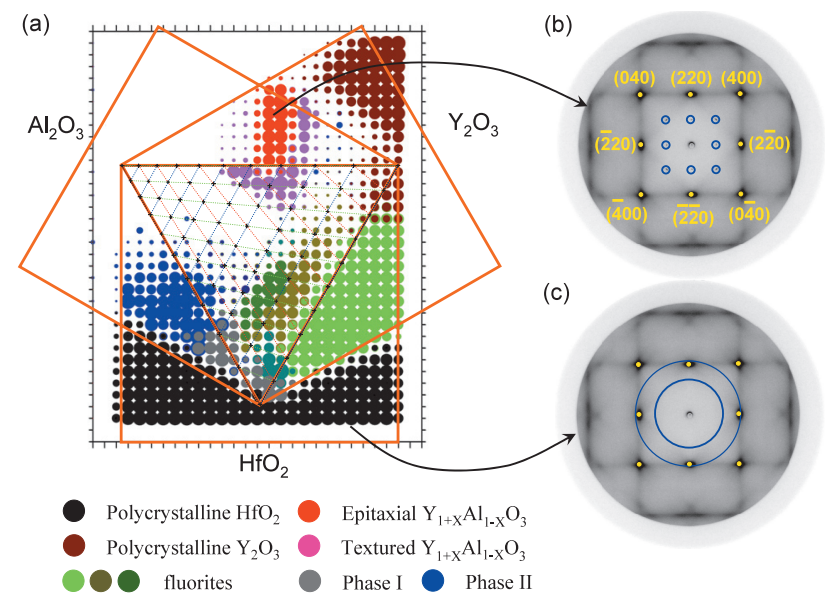

Fig. 5. (a) Reconstruction of the ternary oxide crystal phase diagram for the $\mathrm{Al}_{2} \mathrm{O}_{3}-\mathrm{Y}_{2} \mathrm{O}_{3}-\mathrm{HfO}_{2}$ system deposited on $\mathrm{Si}(100)$ and annealed in $\mathrm{N}_{2}$ at $1000^{\circ} \mathrm{C}$. (b) The two-dimensional Laue diffraction spectrum(plate)at the area of the epitaxial $\mathrm{Y}_{1}+\mathrm{xAl}_{1}-\mathrm{xO}_{3}$ epitaxial phase. The plate contains diffraction signal from the Si substrate (indexed dots), and the diffraction spots from the unidentified commensurate film. (c) The image plate containing diffraction rings taken at the polycrystalline $\mathrm{HfO}_{2}$ area.

amounts of spectroscopic data that cannot be efficiently processed by conventional computers. The power of the rotating anode X-ray source was maintained at $40 \mathrm{KV} \times 400 \mathrm{~mA}=16 \mathrm{KW}$.

For each pixel the two-dimensional XRD spectrums (frames) were collected in the range of $25^{\circ}<$ $2 \theta<55^{\circ}$, and approximately $-126^{\circ}<\chi_{\mathrm{g}}<-54^{\circ} .{ }^{13)}$ The acquisition time for each frame was $60 \mathrm{~s}$. To obtain conventional diffraction intensity $-2 \theta$ scans the frames were further integrated along $\chi_{\mathrm{g}}$ angle on the intervals $\left[-122^{\circ},-58^{\circ}\right],\left[-122^{\circ},-114^{\circ}\right],\left[-66^{\circ}\right.$, $\left.-58^{\circ}\right]$, and $\left[-92^{\circ},-88^{\circ}\right]$. Integration along the $\chi_{\mathrm{g}}$ angle is equivalent to the summation of the diffraction signal from a section of the Debye diffraction rings. The $\left[-122^{\circ},-58^{\circ}\right]$ interval represents approximately $\frac{64}{360} \times 100 \% \approx 17.8 \%$ fraction of the full ring, etc.

The diffraction intensity $-2 \theta$ spectrums obtained by the integration of the two-dimensional frames were used for generation of the XY maps. Series of these maps were generated for each $2 \theta$ angle from 30 to $50^{\circ}$ with a step of $0.1^{\circ}$. A single XY diffraction intensity map taken at a particular $2 \theta$ angle shows which pixels (and also which compositions in the thin film composition spread) contain crystallites with a given lattice spacing corresponding to this particular $2 \theta$ angle. Full set of these XY-diffraction intensity maps yields complete information on the crys- 
tal structures in the spread.

The evolution of the crystal structure along several tie lines or (compositional) trajectories on the ternary composition spread was studied by plotting the conventional XRD intensity $-2 \theta$ scans with respect to the composition change. These scans were obtained from two-dimensional frames by integration along $\chi_{\mathrm{g}}$ angle on the $\left[-122^{\circ},-58^{\circ}\right]$ interval. Finally, XY maps for each crystal phase were generated. For these maps diffraction intensity peaks from a particular phase were integrated on a certain $2 \theta$ and $\chi_{\mathrm{g}}$ interval to allow for the change of the lattice parameter.

\section{3 Combinatorial-HEXRD}

We are currently developing an alternative method for obtaining crystal phase information, which employs high energy synchrotron radiation. The highenergy X-ray beam penetrates thick samples without substantial absorption and reduction of intensity. Therefore, the X-ray diffraction experiments can be performed in a transition mode. High energy X-ray diffraction (HEXRD) yields Laue diffraction patterns similar to those in the transmission electron microscopy (TEM). ${ }^{14)}$ Therefore, the new method provides the benefits of the combinatorial high-throughput scanning TEM. The greatest advantage is the fact that this method is quick and nondestructive; it allows multiple measurements without laborious preparation of the TEM samples. It has high spatial resolution, sufficient for fine compositional resolution of the composition spreads. ${ }^{15}$ )

The high energy X-ray diffraction experiments were performed at the European Synchrotron Radiation Facility (ESRF) at the beamline ID15B (energy of the X-rays $65.7 \mathrm{KeV}$ ). The experimental setup used in this work is sketched in Fig. 2. A monochromatic beam with a cross section of $0.5 \mathrm{~mm} \times 0.5 \mathrm{~mm}$ transmits a monitor diode before entering a custom built vacuum chamber with a crystalline $\mathrm{Si}$ window. During the experiments the chamber was evacuated to a pressure of $10^{-6}$ mbar in order to reduce the background from air scattering. The parasitic scattering from the entrance window was removed by an adjustable pinhole in front of the sample. The transmitted beam exits the chamber via a $2 \mathrm{~mm}$-thick aluminum plate. This exit window removes the lowenergy fluorescence background scattering from the sample. While the direct transmitted beam is absorbed by a lead beam stop mounted directly onto the Al window, the diffracted radiation is recorded by a two-dimensional online image plate reader (mar 345) with $2300 \times 2300$ pixels at a pixel size of $150 \mu \mathrm{m} .{ }^{16)}$
Inside the vacuum chamber, the $\mathrm{X}$-ray beam is passing the composition spread samples mounted on an XYZ-scanning stage. The orientation of the sample relative to the incoming $\mathrm{X}$-ray beam is adjusted by two motorized tilt angles $\phi$ and $\chi$. In addition, the sample assembly could be rotated in the beam by $=$ $\pm 52^{\circ}$. The sample to detector distance $\mathrm{D}=850 \mathrm{~mm}$ was determined from the (200) and (220) Si diffraction spots recorded from a standard $\mathrm{Si}$ wafer.

The image plate detector records a monochromatic Laue diffraction pattern. The wavelength of the synchrotron radiation $\lambda$ is very small, so the corresponding Ewald sphere (ES) has very large radius $R=1 / \lambda$. For this reason ES contains signals from the zero order Laue zone only. As an approximation, the image from the plate represents a plane section of the reciprocal space. In case of the single crystal sample, reconstruction of the 3-dimentional reciprocal space around the origin (000) requires rotation of the sample and collecting the signal from all possible diffraction planes. For polycrystalline samples transmission Laue pattern is a set of concentric circles corresponding to certain lattice plane distances; this suffice for unambiguous crystal phase identification.

\section{Results and Discussion}

\section{1 Investigations of the inert interfaces}

We first consider inert oxide-oxide interfaces as the case study. Reconstruction of the ternary crystal phase diagram for the $\mathrm{NiO}-\mathrm{Co}_{3} \mathrm{O}_{4}-\mathrm{Mn}_{2} \mathrm{O}_{3}$ system deposited onto $\mathrm{SrTiO}_{3}(100)$ is shown in Fig. 3 (a). This visual representation is a color 'bubble plot'. Here, the size of a solid square corresponds to an approximate amount of a particular crystal phase in the composition spread thin film. The elemental oxides at the corners of the fabricated composition spread correspond to the $\mathrm{NiO}$ (rocksalt), $\mathrm{Mn}_{2} \mathrm{O}_{3}$ (bixbyite), and $\mathrm{Co}_{3} \mathrm{O}_{4}$ (spinel) compounds. The crystal structure of the elemental oxide films was the same as that of the bulk ceramic targets used during the film fabrication. The texture or the degree of orientation with respect to the substrate for these three oxides was considerably different. The $\mathrm{NiO}$ phase forms a high-quality epitaxial film. Orientation of the $\mathrm{NiO}$ phase is Rocksalt (100) $\|\left(1 / 2 \frac{1}{2} 20\right) \mathrm{SrTiO}_{3}$. In other words, (100) NiO $\|$ (100) $\mathrm{TiO}_{2}$-terminated surface of the $\mathrm{SrTiO}_{3}$. The $45^{\circ}$-rotation of the rocksalt unit cell relative to the substrate is a common feature for the large misfit rocksalt-perovskite systems. ${ }^{17)}$ The $\mathrm{Co}_{3} \mathrm{O}_{4}$ spinel is strongly textured or practically epitaxial. Orientation of the epitaxial spinels is also commensurate to the 
TiO-terminated plane. However, the quality of epitaxy for the $\mathrm{Co}_{3} \mathrm{O}_{4}$ is much poorer in comparison to that of $\mathrm{NiO}$. The $\mathrm{Mn}_{2} \mathrm{O}_{3}$ bixbyite phase is polycrystalline; it has broad and very weak diffraction rings suggesting strongly disordered phase with small coherent domain size of $\sim 20 \mathrm{~nm}$.

As shown in Figs. 3(b) and 3(c), in the twodimentional XRD frames, the strongly textured or epitaxial films produce diffraction spots, whereas polycrystalline phases show continuous diffraction rings. Since only a fraction of the diffraction ring is recorded in a frame, it is possible to omit the diffraction spots all together. Thus, orientation of the composition spread specimen with respect to the incident $\mathrm{X}$-ray beam (expressed by the $\phi$ angle) must be adjusted to make sure that at least one of the diffraction spots from a particular epitaxial or textured phase appears in the frame. To generate crystal phase map from epitaxial films, the (400) peak of the rocksalt was separated in each frame by firstly integrating the XRD frames along $\chi_{\mathrm{g}}$ angle in the interval between $-92^{\circ}$ and $-88^{\circ}$, then the diffraction intensity $-2 \theta$ scans were integrated between $42.2^{\circ}$ and $43.7^{\circ}$. The presence of the (311) peak of the epitaxial spinel phase was obtained by analyzing $2 \theta$ scans obtained after integration of the frames in the intervals $\left[-122^{\circ}\right.$, $\left.-114^{\circ}\right]$ and $\left[-66^{\circ},-58^{\circ}\right]$. These scans were further integrated along $35.4^{\circ}<2 \theta<37.4^{\circ}$ interval.

Examined composition spread thin film contains commensurate rocksalt $\mathrm{NiO}$ phase doped with $\mathrm{Co}$ and $\mathrm{Mn}$ oxides. The quality of epitaxial growth in this phase strongly depends on the doping. Statistical analysis of the combinatorial XRD is a very useful tool for establishing the best conditions for epitaxial growth. We analyzed the XRD spectrum taken at each point where rocksalt phase exists. Its lattice parameter varies depending on the doping. We separated polycrystalline and epitaxial contributions and plotted intensity of epitaxial component vs. lattice mismatch with $\mathrm{SrTiO}_{3}$ substrate. Figure 4 shows the statistical distribution of such intensity for totally $18 \times 18=324$ compositions.

This figure shows that the materials which have approximately $6.5 \%$ lattice mismatch with the substrate are most likely to yield highest-quality epitaxial film. This result has a statistical importance: the required mismatch will not guarantee good epitaxy because there are many points, which yield lowintensity epitaxial component. However, the $6.5 \%$ mismatch is a necessary condition for good epitaxy. Figure 4 is not completely symmetric, therefore de-

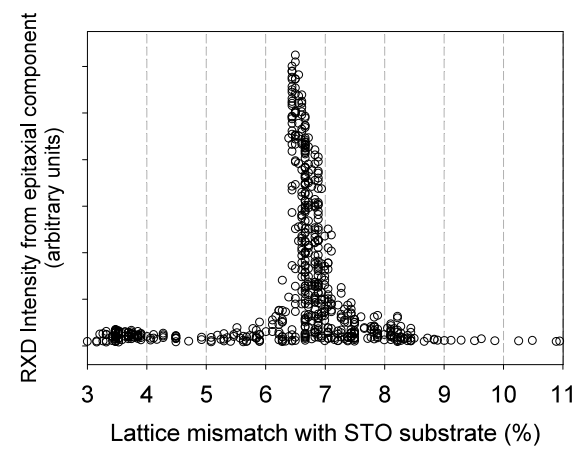

Fig. 4. The strength of the XRD signal from the epitaxial rocksalt vs. lattice mismatch with $\mathrm{SrTiO}_{3}$ substrate. The material having $\sim 6.5 \%$ lattice mismatch yields the highest-quality epitaxial film.

pendence of the epitaxial growth on the rocksalt lattice parameter has a particular threshold value: the mismatch of larger than $6.5 \%$ is more advantageous.

This result is explained as follows. Lattice mismatch of $6.5 \%$ implies a line dislocation density where exactly 1 dislocation appears per 8 unit cells of rocksalt. In other words, one out of 8 unit cells at the oxide-oxide interface should be missing. Most likely, for most of the compositions, the line dislocations at the interface do not form. Instead, they are distributed into separate point defects, specifically, oxygen vacancies. The optimal $6.5 \%$ lattice mismatch corresponds to the defect density such that one out of four oxygen anions at the interface between the rocksalt and perovskite is missing. This is exact condition required to achieve the charge neutrality of the interface.

Statistical analysis shows that for a heteroepitaxial growth, lattice mismatch could be an advantageous factor. This mismatch corresponds to the charge neutrality conditions and strongly depends on the crystal structure of the bonding materials. Our extensive experimental investigations on other oxide systems revealed that this is a general result that applies to all heteroepitaxial oxide-oxide interfaces which exhibit no chemical interaction between the film and the substrate. Epitaxial growth on reactive substrates follows different rules, which will be discussed in the next section.

\section{2 Investigations of the reactive interfaces}

We now consider the more complicated case of the chemically reactive interfaces using the $\mathrm{Re}_{2} \mathrm{O}_{3}-\mathrm{Al}_{2} \mathrm{O}_{3}$ $\mathrm{HfO}_{2}(\mathrm{Re}=\mathrm{La}, \mathrm{Ce}, \mathrm{Y})$ systems on silicon and combinatorial High-Energy X-ray diffraction. These systems include prospective materials for the high- $k$ oxides for transistor gates. In essence, the X-ray beam 
was scanned across the ternary composition spread sample, and crystal phase information at each point was collected and analyzed. Phase maps of the ternary systems were created, and using these maps the new oxide phases were proposed for industrial applications. Figure 5 (a) shows reconstruction of the ternary crystal phase diagram for the $\mathrm{Y}_{2} \mathrm{O}_{3}-\mathrm{HfO}_{2}-\mathrm{Al}_{2} \mathrm{O}_{3}$ system deposited onto $\mathrm{Si}(100)$ substrate and annealed in nitrogen at $1000^{\circ} \mathrm{C}$ for $5 \mathrm{~s}$. As before, the size of the solid circle in this bubble plot represents the amount of a particular crystal phase in the composition spread thin film.

The Combi-HEXRD analysis shows that this ternary spread film contains for the most part polycrystalline phases and their mixtures. Part of the composition spread around $\mathrm{Al}_{2} \mathrm{O}_{3}$ corner contains amorphous or poorly crystallized phases invisible for the $\mathrm{X}$-rays. These compositions are shown on the phase diagram as empty spaces. The elemental oxides at the other two corners of the composition spread are monoclinic $\mathrm{HfO}_{2}$ and cubic $\mathrm{Y}_{2} \mathrm{O}_{3}$. Both of these phases are polycrystalline. The broad binary area along $\mathrm{HfO}_{2}-\mathrm{Y}_{2} \mathrm{O}_{3}$ tie line is a solid solution with perfect cubic fluorite crystal structure. The tetragonal crystal structure designated as 'Phase I' in the Fig. 5 (a) (space group $\mathrm{P} 42 / \mathrm{nmc}$ ) is a derivative of the low temperature phase found in alloys $\mathrm{Al}_{0.08} \mathrm{Zr}_{0.92} \mathrm{O}_{1.96}-$ $\mathrm{Al}_{0.52} \mathrm{Zr}_{0.48} \mathrm{O}_{1.72}$ (PDF cards \#\# 00-053-0549, 00-0530294). 'Phase II' is very poorly crystallized phase related to rhombohedral $\mathrm{Al}_{2} \mathrm{O}_{3}$ (space group $\mathrm{R}-3 \mathrm{c}$ ). Other recorded phases are different derivatives of the fluorite and distorted $\mathrm{HfO}_{2}$ monoclinic crystals with varied lattice constant.

We have discovered that the composition spread contains a narrow epitaxial area around composition $\mathrm{Y}_{1+\mathrm{x}} \mathrm{Al}_{1-\mathrm{x}} \mathrm{O}_{3}$. The new phase has unknown crystal structure. We presently continue and its identification. The composition spread also has patches of the highly-oriented films which are mixtures of epitaxial component and other phases. Figures 5(b) and 5(c), show typical diffraction images (plates) obtained by HEXRD. The polycrystalline phases produce full Laue diffraction rings, whereas the strongly textured or epitaxial films produce diffraction spots. The most intense spots correspond to the diffraction signal from the silicon substrate. Laue diffraction image taken by this technique is basically a plane section of the reciprocal space of the crystal that necessarily crosses the origin point (000). Unambiguous phase identification of the single crystal requires a 3-dimentional reconstruction of the reciprocal space around the ori- gin. To make this reconstruction one must rotate the specimen with respect to the incident X-ray beam and collect all possible 2-dimensional sections. This is impossible during combinatorial screening. Therefore, the definitive determination of the crystal parameters of the new epitaxial phase is yet impossible.

We found that the originally deposited composition spread thin films never form the epitaxial phase by itself. The specimens must be exposed to thermal treatment above approximately $950^{\circ} \mathrm{C}$ for the new phase to form. Thus, the chemical reaction with the substrate is critical. TEM investigations suggest that the new oxide rapidly reacts with $\mathrm{Si}$, but the interface remain sharp and clean because the oxide sponges up the byproducts of this reaction. Therefore the interfacial integrity may be not thermodynamic, but kinetic in nature. Absorbing of the reaction products by the precursor film has recently been demonstrated for some oxide substrates, for example in a process called reactive solid-phase epitaxy. ${ }^{18)}$ The epitaxial phase, which readily forms on the $\mathrm{Si}(100)$ substrate, does not appear on $\mathrm{Si}(111)$. This implies that silicon diffusivity in the new epitaxial oxide is strongly direction-dependent.

Rare earth aluminate alloys, $\mathrm{Re}_{2} \mathrm{O}_{3}-\mathrm{Al}_{2} \mathrm{O}_{3}$, generally exhibit substantial solubility of $\mathrm{SiO}_{2}{ }^{19)}$ For example, the quasibinary system $\mathrm{Gd}_{4} \mathrm{Al}_{2} \mathrm{O}_{9}$ shows extended silicon dioxide solubility (up to $\sim 18 \mathrm{~mol} \%$ $\left.\mathrm{SiO}_{2}\right) .{ }^{20}$ ) The diffusion rate of the reaction product in the precursor material must have been very high. The comprehensive information on the diffusion rates is lacking for most of the aluminate material systems. However, relatively low eutectic temperatures for the ternary $\mathrm{Re}_{2} \mathrm{O}_{3}-\mathrm{Al}_{2} \mathrm{O}_{3}-\mathrm{SiO}_{2}$ systems (they lie between $1200^{\circ} \mathrm{C}$ for $\mathrm{La}$ and $1435^{\circ} \mathrm{C}$ for $\mathrm{Yb}$ ) also support our findings. Also, melting temperature of a given ternary $\mathrm{Re}_{2} \mathrm{O}_{3}-\mathrm{Al}_{2} \mathrm{O}_{3}-\mathrm{SiO}_{2}$ composition is usually much lower than that of the $\mathrm{Re}_{2} \mathrm{O}_{3}-\mathrm{Al}_{2} \mathrm{O}_{3}$ precursor. I.e. addition of $\mathrm{SiO}_{2}$ sharply increases further diffusion of silicon in the $\mathrm{Re}_{2} \mathrm{O}_{3}-\mathrm{Al}_{2} \mathrm{O}_{3}$ precursor.

Reactive oxides can form commensurate films on silicon by molecular beam epitaxy. ${ }^{21)}$ However, high temperature interfacial reaction destroys epitaxial connection, and the films become polycrystalline. Here we show that the material able to absorption of reaction products and interfacial self-organization could form epitaxial films after a thermal treatment. Therefore, the clue to the epitaxy involving the reactive interfaces is careful balancing of the chemical reaction and diffusion. 


\section{Conclusions}

The heteroepitaxial interfaces can be divided into two general categories: the inert and chemically reactive boundaries. We have described systematic combinatorial exploration of heteroepitaxial thin films using novel XRD and HEXRD techniques. We showed that for the inert interfaces the lattice mismatch is the dominating factor in the epitaxial growth. The most beneficial mismatch depends on the crystal characteristics of both the substrate and the film. For example for the rocksalt-perovskite interface it must be equal to $6.5 \%$. For the reactive interfaces the chemical and kinetic parameters of the interaction play the most important role. In order to achieve the stable interface and the self-restoring epitaxy, the diffusion of the reaction products away from the interface is required. When outdiffusion is achieved, the lattice mismatch factor must be taken into account in order to obtain the best-quality crystalline films.

\section{Acknowledgements}

We thank V. Honkimäki, T. Buslaps and $\mathrm{J}$. Okasinski at the ESRF beamline for help, and $\mathrm{H}$. Reichert for discussions. This work has been funded by National Institute for Materials Science (Japan) in the project 'Combinatorial Materials Exploration and Technology' and by the 'Core to Core Program' of the Japan Society for Promotion of Science.

\section{References}

1) J. Goldberger, R. He, Y. Zhang, S. Lee, H. Yan, H.J. Choi and P. Yang: Nature 422, 599 (2003).

2) S. Nakamura, G. Fasol and S.J. Pearton: "The Blue Laser Diode: The Complete Story" (Springer Verlag, New York, 2000).
3) E. Ascher, H. Rieder, H. Schmid and H. Stossel: J. Appl. Phys. 37, 1404 (1966).

4) J.-H. Li, L. Chen, V. Nagarajan, R. Ramesh and A.L. Roytburda: Appl. Phys. Lett. 84, 2626 (2004).

5) D.A. Muller: Nature Materials 4, 645 (2005).

6) A.I. Kingon, J.-P. Maria and S.K. Streiffer: Nature 406, $1032(2000)$.

7) A.A. Demkov and A. Navrotsky: "Materials Fundamentals of Gate Dielectrics" (Springer, New York, 2005).

8) R.M. Wallace and G. Wilk: Mater. Res. Bulletin 27, 192 (2002).

9) H. Koinuma and I. Takeuchi: Nature Materials 3, 429 (2004).

10) K. Hasegawa, P. Ahmet, N. Okazaki, T. Hasegawa, K. Fujimoto, M. Watanabe, T. Chikyow and H. Koinuma: Appl. Surf. Sci. 223, 229 (2004).

11) Y. Yamamoto, R. Takahashi, Y. Matsumoto, T. Chikyow and H. Koinuma: Appl. Surf. Sci. 223, 9 (2004).

12) D.A. Kukuruznyak, P. Ahmet, A. Yamamoto, F. Ohuchi and T. Chikyow: J. Appl. Phys. 98, 043710-1-4 (2005).

13) B.B. He: Third International Conference on Experimental Mechanics, Proc. SPIE 4537, 127 (2002).

14) Z. Ren and K. Moffat: J. Appl. Crystallogr. 28, 461 (1995).

15) H. Reichert, V.N. Bugaev, O. Shchyglo, A.Schöps, Y. Sikula and H. Dosch: Phys. Rev. Lett. 87, 236105 (2001).

16) M. Mezger, H. Reichert, I.B. Ramsteiner, A. Udyansky, O. Shchyglo, V.N. Bugaev, H. Dosch and V. Honkimäki: Phys. Rev. B 73, 184206 (2006).

17) G. Chern: Surf. Sci. 387, 183 (1997).

18) H. Ohta, K. Nomura, M. Orita, M. Horano, K. Ueda, T. Suzuki, Y. Ikuhara and H. Hosono: Adv. Funct. Mater. 13, 139 (2003).

19) U. Kolitsch, H.J. Seifert and F. Aldinger: J. Phase Equilib. 19, 426 (1998).

20) U. Kolitsch, H.J. Seifert and F. Aldinger: J. Alloys and Comp. 257, 104 (1997).

21) R.A. McKee, F.J. Walker and M.F. Chisholm: Science 293, 468 (2001). 